\title{
A Possible Solution to the Disagreement about the Hubble Constant
}

\author{
Frank R. Tangherlini \\ San Diego, CA, USA \\ Email: frtan96@gmail.com
}

How to cite this paper: Tangherlini, F.R. (2018) A Possible Solution to the Disagreement about the Hubble Constant. Journal of Modern Physics, 9, 1827-1837. https://doi.org/10.4236/jmp.2018.99116

Received: July 19, 2018

Accepted: August 13, 2018

Published: August 16, 2018

Copyright $\odot 2018$ by author and Scientific Research Publishing Inc. This work is licensed under the Creative Commons Attribution International License (CC BY 4.0).

http://creativecommons.org/licenses/by/4.0/

\begin{abstract}
The current disagreement about the Hubble constant $H_{0}$ was described as a "Crisis in Cosmology", at the April (2018) Meeting of the American Physical Society, and hence its resolution is of utmost importance. This work proposes that the solution to the disagreement between the Planck Collaboration cosmic microwave background (CMB) value of $H_{0}$, together with the very close BOSS Collaboration baryon acoustic oscillation (BAO) value, and the significantly higher value of $H_{0}$ found by the SHOES Collaboration cosmic distance ladder (CDL) work, is due to the fact that the $\mathrm{CMB}$ and $\mathrm{BAO}$ values of $H_{0}$ are not for an accelerating universe, as generally believed, but are actually the values for a decelerating universe. In contrast, the CDL value of $H_{0}$ is indeed that for an accelerating universe. It is shown that by replacing the negative deceleration parameter in the expression for $\log H_{0}$ in the CDL work by a positive deceleration parameter, the value of $H_{0}$ can be brought down to agree with the CMB and BAO lower values. There is a brief review of the author's decelerating model based on the Einstein de Sitter universe, augmented by a model of dark energy that does not have a negative pressure, but instead has a non-dispersive index of refraction $n$, causing the speed of light through the dark energy of intergalactic space to be reduced to $c / n$. As reported earlier, this assumption is sufficient to accommodate the increase in apparent magnitude of the Type Ia supernovae (SNe Ia). Additional support for the model is presented, together with a proposal for astronomical falsification.
\end{abstract}

\section{Keywords}

Hubble Constant Disagreement, CMB \& BAO Determinations, Decelerating Interpretation, CDL Determination \& Modification

\section{Introduction}

The current disagreement about the Hubble constant $H_{0}$ is widely recognized as 
a serious problem in cosmology, indeed, as indicated in the Abstract, it has been recently described as a "Crisis in Cosmology." The purpose of this work is to show that there is a possible solution to the problem based on the author's proposal in [1], and further developed in [2] [3] [4] [5], that the universe is not accelerating, but is actually decelerating. In Section 2, for the reader's convenience, this alternative model that involves a reduction in the speed of light through the dark energy of intergalactic space (IGS) will be briefly reviewed. In Section 3, it will be proposed that the disagreement between the Planck collaboration [6] $\mathrm{CMB}$ value $H_{0}=67.90 \pm 0.55 \mathrm{~km} \cdot \mathrm{s}^{-1} \cdot \mathrm{Mp} \cdot \mathrm{c}^{-1}$, that utilizes the $\Lambda \mathrm{CDM}$ model, and the SHOES collaboration [7] cosmic distance ladder (CDL) value, $H_{0}=73.24 \pm 1.74 \mathrm{~km} \cdot \mathrm{s}^{-1} \cdot \mathrm{Mp} \cdot \mathrm{c}^{-1}$, is due to the CMB value for $H_{0}$ actually being that for a decelerating universe, whereas the CDL value for $H_{0}$ is indeed, as presented, that for an accelerating universe. The BOSS collaboration [8] BAO value, $H_{0}=67.6 \pm 0.5 \mathrm{~km} \cdot \mathrm{s}^{-1} \cdot \mathrm{Mp} \cdot \mathrm{c}^{-1}$, is so close to the CMB value, and because it is simpler to deal with analytically, it will be used to justify the decelerating interpretation of the lower $\mathrm{CMB}$ and $\mathrm{BAO}$ values. It will also be shown that when the CDL expression for $\log H_{0}$, given in [7], that uses a negative deceleration parameter in its kinematic term, $q_{0}=-0.55$, is changed to a positive deceleration parameter, $q_{0}=0.50$, which is appropriate for the decelerating Einstein de Sitter (EdS) universe that is taken as reference, the ratio of the Hubble constant for the accelerating universe to the Hubble constant for the decelerating universe is reduced. In fact, it is shown that for reasonable values of redshifts, the ratio of the two Hubble constants is just the ratio of two kinematic terms that in turn become equal to $H_{0}(C D L) / H_{0}(B A O)$, or $H_{0}(C D L) / H_{0}(C M B)$. Although the EdS universe was used as a reference decelerating universe, it was shown in [3] that, for $\Omega_{\text {tot }}$ sufficiently close to unity, a closed universe might alternatively be used. It is also pointed out there that the closed universe yields an upper bound on $\Lambda$ that is too small to accommodate the accelerating universe, and if the EdS universe can be seen as the limit of a closed universe with infinite radius, then the upper bound on $\Lambda$ goes to zero.

Since the above results do not necessarily prove that the universe is decelerating, but only make it reasonable, in Section 4, two tests of the decelerating model, that have been discussed earlier, particularly in [4] [5], are briefly reviewed. The first test is based on the search for neutrinos correlated with gamma ray bursts (GRBs), and the second test is based on the possibility of alternative galactic sources for discordant redshifts. In Section 5, there are conclusions.

\section{Brief Review of the Decelerating Model}

In the decelerating model, it is assumed that the dark energy of intergalactic space (IGS), instead of having a negative pressure, that causes the expansion of the universe to be presently accelerating, has rather an index of refraction $n$, so that the speed of light through IGS is reduced to $c / n$. A least squares fit of the EdS universe to the accelerating $\Lambda \mathrm{CDM}$ universe, that is presently favored to ex- 
plain the increased apparent magnitude of the Type Ia supernovae (SNe Ia), found by Riess et al. [9], Schmidt et al. [10], and Pearlmutter et al. [11], yielded $n \approx 1.5$. It is further assumed that there is no dispersion across the entire electromagnetic spectrum, based on the original work in the optical range [9] [10] [11], and later investigations covering a wide range of the spectrum by Costa $e t$ al. [12], Metzger et al. [13], and Frail et al. [14]. However, within the galaxies themselves, the speed of light remains the vacuum speed $c$. Since Riess et al. [15] found that at $z=1.65 \pm 0.15$ there was no acceleration, it is assumed in this model that before this value of redshift there is negligible dark energy, and there is only dark matter in IGS as well as in the galaxies. However, as the universe expanded and reached the above value of redshift, it is further assumed that because of expansion cooling, the dark matter in IGS underwent a phase transition into dark energy, characterized by the development of an index of refraction that eventually became the above value, $n \approx 1.5$. Only the dark matter in IGS undergoes the phase transition, because it is only IGS that expands, not the galaxies themselves. At the ill-defined boundaries of the galaxies there will be a transition region for $n$ which will be ignored in this preliminary, highly-simplified model. It is outside of the scope of this work to attempt to hypothesize on the nature of the particle or particles that constitute dark matter, since it is reasonable that one should first determine whether this phenomenological model is valid. The least squares fit for $n$ in the author's first three papers [1] [2] [3] assumed the phase transition was completed by $z=1.0$, so that $n$ was taken as constant from $z=1.0$ to $z=0$. However, subsequent work [4] [5] suggested that $n$ became constant somewhat later, possibly at $z \approx 0.6$. The reduced speed of light through IGS allows the EdS universe the additional time to expand to its size at the present epoch, so as to give rise to the increase in distance that is needed to explain the increased apparent magnitude of the SNe Ia. It was shown in [1] that the increase in apparent magnitude, $\delta m$, under the assumption $n$ is a constant over the range of redshifts under consideration, is given by

$$
\delta m=5 \log (1+(n-1) \ln (1+z))
$$

while the corresponding logarithmic fractional increase in distance $d$ is given by

$$
d=\log (1+(n-1) \ln (1+z))
$$

The above value for $d$ is used when comparing the logarithmic distance to the standard ruler of the BAO that is fitted by the $\Lambda \mathrm{CDM}$ model, with that fitted by the EdS model that has been augmented by $d$; see, e.g., Equation (16) in [1], and also (3) below, after putting it into logarithmic form. Since $\delta m=5 d$, the percentage of disagreement for comparison of the model with the $\Lambda \mathrm{CDM}$ fit to the SNe Ia should be the same as the percentage of disagreement for the comparison of the model with the $\Lambda \mathrm{CDM}$ fit to the BAO. This was shown in [1] to be the case by using the tables of comparison given there.

\section{Application to the Hubble Constant Disagreement}

The resolution of the disagreement about the Hubble constant $H_{0}$ that is pro- 
posed here begins with the observation that the $\mathrm{CMB}$ value not only disagrees with the CDL value, but is significantly lower than the $C D L$ value. This would be the case if the $\mathrm{CMB}$ value of $H_{0}$ were not for an accelerating universe, but were actually for a decelerating universe. In contrast, the CDL value of $H_{0}$ is certainly for an accelerating universe, because it explicitly involves the deceleration parameter $q_{o}$ to which is assigned the value $q_{0}=-0.55$ [7]. The proposed alternative interpretation of the $\mathrm{CMB}$ value for $H_{0}$ arises from the observation that since $H_{0} \equiv \dot{a}_{0} / a_{0}$, where $\mathrm{a}(\mathrm{t})$ is the expansion parameter for the flat Friedmann LeMaittre Robertson Walker (FLRW) line element $\mathrm{d} s^{2}=c^{2} \mathrm{~d} t^{2}-a(t)^{2} \mathrm{~d} r^{2}+r^{2}\left(\mathrm{~d} \theta^{2}+\sin ^{2} \theta \mathrm{d} \phi^{2}\right)$, and since $a_{0}$ is necessarily the same for both models, and also since $\dot{a}_{0}($ accel. $)>\dot{a}_{0}($ decel. $)$, then $H_{0}($ accel.$)>H_{0}($ decel . $)$. The inequality for the expansion parameter velocities arises in the following way: In the very early universe, before the accelerating term became significant, both models would be expanding at essentially the same rate, but as the effect of the accelerating term became larger, the $\Lambda \mathrm{CDM}$ universe's deceleration became increasingly less than that of the EdS universe, and eventually the accelerating term became dominant, and caused the universe to accelerate, so that by the times (which are different, less for the $\Lambda \mathrm{CDM}$ universe) each universe has expanded to $a_{0}$ the above inequality for the Hubble constants resulted. To be sure, the above qualitative analysis does not guarantee that $H_{0}($ accel.$)>H_{0}($ decel. $)$ by the observed amount, but it is suggestive, and it serves to motivate the quantitative analysis given further below. It will be shown there that by replacing in the expression for $H_{0}(C D L)$ a value for the deceleration parameter that is the value for a decelerating universe, so that $q_{0}>0$ instead of $q_{0}<0$ as was used in [7], one can obtain a value of $H_{0}$ that agrees with $H_{0}(B A O)$ which, as noted above, is essentially the same as $H_{0}(C M B)$.

But before carrying out this analysis, it is necessary to show how it could be possible that the reported $\mathrm{CMB}$ and $\mathrm{BAO}$ values of $H_{0}$ could be that for a decelerating universe, since they are presented as being the Hubble constants for the accelerating $\Lambda \mathrm{CDM}$ universe. The key is to note a well-known ambiguity in the term "dark energy." On the one hand, such as in the author's work, one of the interpretations leads to a density parameter for dark energy $\Omega_{d e} \approx 0.7$ that is needed to arrive at $\Omega_{\text {tot }} \approx 1$, since, as is well-known, dark matter and baryonic matter together only account for about $30 \%$ of $\Omega_{\text {tot }}$. This was shown independently of the CMB determination, by Verde et al. [16], and Hawkins et al. [17], based on studies that utilized the $2 \mathrm{dF}$ Galaxy redshift survey. On the other hand, the more frequent use of the term assumes that dark energy is associated with a negative pressure $P$, and in the case of the $\Lambda \mathrm{CDM}$ model, with $P=w U$, in which $w=-1$, and where $U$ is the positive energy density associated with the cosmological term, which necessarily leads to a density parameter of $\sim 0.7$ as well. Thus one has the relation $\Omega_{\Lambda}=\Omega_{d e}$, even though the corresponding energy-stress source tensors used in the Einstein field equations are different. Since the way the CMB workers extract $H_{0}$ from their measurements is rather complicated, and well beyond the scope of this work, it will be shown for the comparable BAO 
determination of the Hubble constant, that $H_{0}(B A O)$ can be alternatively interpreted as being for a decelerating universe that has the same density of dark energy as the accelerating $\Lambda \mathrm{CDM}$ universe, but does not have the negative pressure associated with the cosmological term.

As was shown in [1], one can obtain the same distances that are found in the $\Lambda \mathrm{CDM}$ universe to the "standard ruler" of the BAO by using instead the decelerating EdS universe, augmented by the fractional distance increase provided by the reduced speed of light, the logarithm of which is given in (2). From the analysis in Equation (9) to Equation (18) of [1], one obtains an Equation for the effective distance, $a_{0} r(z)$, that involves the Hubble constant, and that relates the two models, that is given by

$$
c H_{0}^{-1} \mathrm{X}_{\Lambda}(z)=c H_{0}^{-1} \mathrm{X}_{m}(z)(1+(n-1) \ln (1+z))
$$

where $\mathrm{X}_{\Lambda}(z)$ and $\mathrm{X}_{m}(z)$ are dimensionless integrals derived from the field equations for energy densities for the two different universes, and in which each side of the above equation is equal to the effective distance to the standard ruler of the BAO, while $z$ is its redshift. Actually there are two distances that are determined observationally: The angular distance $D_{A}(z)=a_{0} r(z) /(1+z)$, and the longitudinal distance $D_{L}(z)=a_{0} r(z)(1+z)$, so that the effective distance $a_{0} r(z)$ is given by $a_{0} r(z)=\left(D_{A}(z) D_{L}(z)\right)^{1 / 2}$. For the determination of these distances, that are presented as supportive of the flat $\Lambda$ CDM universe, see Anderson et al. [18], and Anderson et al. [19]. The determination of $H_{0}$ from the left hand side of (3) by setting $a_{0} r(z)=c H_{0}^{-1} \mathrm{X}_{\Lambda}(z)$ would seem to yield the Hubble constant for an accelerating universe given by $H_{0}=c \mathrm{X}_{\Lambda} / a_{0} r(z)$, which is the current interpretation. However, one can alternatively regard $a_{0} r(z)$ as having been set equal to the right hand side of (3), and hence it would yield the Hubble constant for a decelerating universe with reduced speed of light, which is the interpretation proposed here. To resolve the conflict in the two interpretations, it will be shown next that when the CDL expression for $H_{0}$ is taken to be that for a decelerating universe, it can be lowered in value to equal the BAO determination.

In [7], from their Equation (9), one has the following expression for $\log H_{0}$

$$
\log H_{0}=\frac{M_{x}^{0}+5 a_{x}+25}{5}
$$

where $M_{x}^{0}$ is a distance modulus, and from Equation (5) in [7] one has that

$$
a_{x}=\log \left(c z\left\{1+\frac{1}{2}\left[1-q_{0}\right] z-\frac{1}{6}\left[1-q_{0}-3 q_{0}^{2}+j_{0}\right] z^{2}+O\left(z^{3}\right)\right\}\right)-0.2 m_{x}^{0}
$$

where $j_{0}=1$ from prior deceleration, and $m_{x}^{0}$ is another distance modulus. It will be convenient to combine the two distance moduli and the additional constant term into the combined distance modulus $M$ defined by

$$
M \equiv \frac{M_{x}^{0}-m_{x}^{0}+25}{5}
$$

and upon introducing the kinematic term $k\left(z, q_{0}, j_{0}\right)$ defined by 


$$
k\left(z, q_{0}, j_{0}\right) \equiv\left\{1+\frac{1}{2}\left[1-q_{0}\right] z-\frac{1}{6}\left[1-q_{0}-3 q_{0}^{2}+j_{0}\right] z^{2}\right\}
$$

in which the correction term $O\left(z^{3}\right)$ has been omitted, one can rewrite $\log H_{0}$ as

$$
\log H_{0}=M+\log (c z)+\log k\left(z, q_{0}, j_{0}\right)
$$

In this form, the distance modulus $M$ has an intuitive interpretation. The original Hubble relation, based on the first order Doppler effect, takes the form $H_{0} D=c z$ where $D$ is the proper distance to the galaxy with recessional speed $c z$. For larger values of redshift, and when acceleration is taken into account, the revised Hubble law takes the form $H_{0} D=c z k\left(z, q_{0}, j_{0}\right)$, upon neglect of higher order terms, but including a period of deceleration represented by $j_{0}$. Hence it follows, upon taking the logarithm of the preceding equation and utilizing (8), that $M=-\log D$. However, the following argument does not make use of the value of $M$, since one is going to subtract from $\log H_{0}$ for an accelerating universe, $\log H_{0}$ for a decelerating universe. To simplify the notation, primes will be used to denote the latter, so that upon subtracting, and suitably rewriting the result, one has

$$
\log \left(\frac{H_{0}}{H_{0}^{\prime}}\right)=M-M^{\prime}+\log \left(\frac{k\left(z, q_{0}, j_{0}\right)}{k\left(z, q_{0}^{\prime}, j_{0}\right)}\right)
$$

After setting $q_{0}=-0.55$ for the accelerating universe as given in [7], and $q_{0}=0.5$ for the decelerating EdS universe, equation (9) takes the form

$$
\log \left(\frac{H_{0}(-0.55)}{H_{0}(0.5)}\right)=M-M^{\prime}+\log \left(\frac{1+0.775 z-0.274 z^{2}}{1+0.25 z-0.125 z^{2}}\right)
$$

One can determine $M$ from (8) as a function of redshift since the value of $H_{0}(-0.55)$ is known, or alternatively from its definition in (6). However, in order to obtain $M^{\prime}$ it will be necessary for the astronomers to re-interpret their data under the assumption that the universe is decelerating, so that one can check whether $H_{0}(0.5)$ is equal to the BAO value of $H_{0}$ after allowing for the measurement uncertainties. In the present case, given the lack of such empirical information for $M^{\prime}$, one can proceed as follows: Set the ratio of the two Hubble constants, which will be denoted by $\eta$, equal to $H_{0}(C D L) / H_{0}(B A O)$; in addition, for simplicity, take their mean values as fiducial, so that one has $\eta=73.24 / 67.6=1.083$. Next, find the behavior of $M-M^{\prime}$ as function of redshift by using (10) with $\eta=1.083$. Since it is mentioned in [7] that the primary fit was based on the redshift range, $0.0233<z<0.15$, it is of interest to determine $M-M^{\prime}$ for, say, $z=0.03$ and $z=0.15$. One finds for these two redshifts, $M-M^{\prime}=0.0323$, and $M-M^{\prime}=0.0084$, respectively. Thus $M-M^{\prime}$ is approaching zero with increasing redshift, for this range of redshifts. The redshift $z^{*}$ for which $M-M^{\prime}=0$ is of special interest, since under these circumstances $\log \eta=k\left(-0.55, z^{*}, j_{0}\right) / k\left(0.5, z^{*}, j_{0}\right)$. Because of the lack of empirical values for $M^{\prime}$ one cannot predict $H_{0}(0.5)$, so that a reasonable alternative is 
to predict the values of $z^{*}$ for which $M-M^{\prime}=0$. From (10), when $M-M^{\prime}=0$, one obtains for $z^{*}$, after removing the logarithms, the quadratic equation

$$
\left(z^{*}\right)^{2}-\frac{0.775-\eta 0.250}{0.274-\eta 0.125} z^{*}+\frac{\eta-1}{0.274-\eta 0.125}=0
$$

For $\eta=1.083$, one has the two roots $z^{*}=0.17$, and $z^{*}=3.47$. Because terms of $O\left(z^{3}\right)$ have been omitted, the higher redshift value is possibly less reliable than the lower value, and in any case this higher value is unacceptable. Since, as indicated in [7], more than 600 SNe Ia were used in a Hubble plot, with redshifts ranging, $0.01<z<0.4$, it follows that the higher value of $z^{\star}$ lies well outside the range of redshifts considered in the CDL work, unlike the lower value. Also, if one takes the fiducial value of $\eta$ to be that for $H_{0}(C D L) / H_{0}(C M B)$ so that $\eta=73.24 / 67.90=1.079$, one finds that the two roots are $z^{*}=0.16$, and $z^{*}=3.47$. Hence the smaller root is clearly more sensitive to the value of $\eta$ than the larger root, which, as indicated above, is to be excluded.

Although the above analysis makes it reasonable that the origin of the disagreement about the Hubble constant is due to the fact that the CMB and the $\mathrm{BAO}$ values are for a decelerating universe, in contrast with the CDL value, which is clearly for an accelerating universe, the analysis does not provide a rigorous proof that the universe is decelerating, since the above lower values of $z^{*}$, when uncertainties are allowed for, have not yet been confirmed empirically, and consequently there is always the possibility, until it is shown otherwise, that the $\mathrm{CMB}$ and $\mathrm{BAO}$ values for $H_{0}$ could be brought up to be in agreement with the CDL value. Therefore, other possible proofs that the universe is decelerating, and that the speed of light in IGS is $\sim 2 c / 3$, are required. In the next section, two further predictions of the model that support this proposed reduction of the speed of light in IGS are described. They have been mentioned earlier in [2] [4] [5], but for completeness, they are briefly presented here, together with some additional comments.

\section{Additional Predictions of the Model}

A prediction that fully supports the model, although it does not confirm it, was first discussed in [2], and in greater detail in [5]. It is based on attempts to find correlation of neutrinos with gamma ray bursts (GRBs). Significantly, to date, none have been found. The most impressive search, in terms of the number of null events, is that of the Boreximo collaboration, as given in Agostino et al. [20]. They searched from 2007-2015 for neutrinos and anti-neutrinos in the energy range, $1.5 \mathrm{MeV}$ - $17 \mathrm{MeV}$, correlated with 2350 observations of GRBs, and found no statistically significant excess above background. Since in the model the speed of light through the dark energy of IGS is $\sim 2 c / 3$, and since for these energies neutrinos travel very close to $c$, if $D$ is a typical distance to the cosmological sources of the GRBs, the time difference for the arrival of the neutrinos and the 
GRBs would be $\sim D / 2 c$, after neglecting the time the GRBs spent traveling through the Milky Way with speed $c$. Since the GRBs come from cosmologically distant sources, $D / 2 c$ is of the order of millions of years, so that even if there were a sufficient fluence of neutrinos to be detectable, the neutrinos would have arrived millions of years earlier than the GRBs. Hence the model predicts that there cannot be any correlation of neutrinos with GRBs, which is what is observed. This has also been shown to be true with numerous other searches as well [21]-[30]. To be sure, it could be the case that none of the GRB sources produce a sufficient fluence of neutrinos to be detectable above the background, which would therefore give rise to the same null result. However, there is a possible counter-example to this alternative: although Artsen et al. [31] reported that the IceCube collaboration had detected two PeV neutrinos without any correlation with GRBs, it is nevertheless possible that a GRB was produced along with these very high energy neutrinos, but the GRB will not arrive until millions of years in the future. Furthermore, as emphasized in [5], if one assumes that none of the GRB sources produced a measureable fluence of neutrinos, that amounts to thousands of assumptions: one assumption for each case of detected GRB unaccompanied by neutrinos. Hence, according to Occam's Razor, this large number of assumptions makes this explanation less preferable than that of the proposed model that uses only one assumption about the reduced speed of light through IGS.

Although the above absence of correlations supports the model, as well as the proposed resolution of the disagreement over the Hubble constant, neither fully confirms it. On the other hand, the following tests based on alternative sources for discordant redshift galaxies would confirm the model, and can be carried out quite readily. It was shown by Bahcall [32] that of the 64 cases of discordant redshifts, compiled and edited by Arp [33], 40 of them could be explained as due to accidental superposition, i.e., the higher redshift galaxy (HRG) being behind the lower redshift galaxy (LRG), at a cosmological distance, and shining through. He concluded that these $\sim 40$ cases were sufficiently close in number to the 64 observed cases that all the cases could be explained as due to accidental superposition. However, upon re-examining Bahcall's estimate, I found that he had rounded-up two of the numbers he had used in the product that led to the $\sim 40$ cases, and that when I did not round up these numbers, the product yielded only 30 cases that could be explained as due to accidental superposition. This suggested that about half the remaining cases are not due to accidental superposition, but are due to something else. It turns out the proposed model can explain the remaining cases, because it predicts that the dark energy of IGS has an index of refraction of $\sim 1.5$, while galaxies have an index of refraction of unity. Consequently, a light ray from an HRG that is not behind the LRG, but off to the side, angle-wise, upon being incident upon a LRG, at a suitable angle relative to the local normal, could be refracted in such way that it would pass through the LRG, and after being further refracted upon exiting the LRG, it could travel along a path that would bring it to the astronomer who would see the HRG as a 
discordant redshift galaxy, while at the same time by suitably redirecting the telescope, depending on the location of the discordant redshift image, the astronomer would see the HRG directly. (Please see Figure 1 in [4].) Since galaxies do not have sharp edges, a light ray will encounter a variable behavior of $n$ upon entering and leaving the LRG, as well as entering the Milky Way, so that the above scenario is highly simplified, but the qualitative picture should be valid. Hence it is a prediction of the model that the proposed HRGs will be found as a supplementary source of discordant redshifts. However, if they should not be found, this would not necessarily falsify the model, since it could turn out that the numbers Bahcall proposed did not accurately describe the situation. On the other hand, the model would definitely be falsified if it were found that an HRG was suitably located off to the side of a LRG in such a way that it should have given rise to a discordant redshift but did not, since that would mean the ray from the HRG had most likely gone through the LRG with negligible refraction, and would thereby demonstrate that IGS does not have an observably higher index of refraction than the LRG, in conflict with the model.

\section{Conclusion}

The above work has shown that one can resolve the current disagreement about the Hubble constant by assuming that the $\mathrm{CMB}$ and $\mathrm{BAO}$ determinations of $H_{0}$ are not for the accelerating $\Lambda \mathrm{CDM}$ universe, but for a decelerating EdS universe, in which the density parameter for the dark energy in the EdS universe satisfies the relation $\Omega_{d e}=\Omega_{\Lambda}$. However, unlike the cosmological term, the dark energy in this model does not have a negative pressure associated with it, but instead it has an index of refraction $n$. As a test of this proposal, it was shown that the CDL's higher value for $H_{0}$ could be lowered to agree with the CMB and BAO determinations by re-evaluating the CDL value of $H_{0}$ for a decelerating universe, rather than for an accelerating universe. In order to obtain the additional distance in the EdS decelerating universe that is needed to explain the increased apparent magnitude of the SNe Ia that led astronomers [9] [10] [11] to infer that the universe is accelerating, it is necessary to assume the speed of light through the dark energy of IGS is reduced to $c / n$, where $n \approx 1.5$. This assumption gives rise to a challenging problem: How can such a very low density substance as the dark energy have an index of refraction comparable to some types of glass, and moreover do it without introducing any dispersion? Furthermore, since it has been shown recently from a binary neutron star merger that the resulting gravitational waves (GWs) Abbott et al. [34] [35] arrive at essentially the same time $(\sim 1.7 \mathrm{~s})$ as the GRBs Goldstein et al. [36] and Savchenko et al. [37], this index of refraction for electromagnetic radiation would have to hold for gravitational waves as well! Consistent with this, the Borexino collaboration, as reported in d'Agostino et al. [38], did not find any neutrinos above background correlated with the arrival of GWs. The only explanation that seems to embrace all these findings, as remarked in [5], is that one is possibly encountering consequences of that long-sought unified theory of electromagnetism and gravitation that 
might predict how dark energy could influence the propagation of EM radiation and GWs in this way. However, before one can conclude that dark energy does indeed have such extraordinary properties, it is essential to ascertain whether the above predictions concerning the Hubble constant and discordant redshifts actually hold. Hopefully this work, as well as those that preceded it, will encourage astronomers to undertake such investigations.

\section{Conflicts of Interest}

The authors declare no conflicts of interest regarding the publication of this paper.

\section{References}

[1] Tangherlini, F.R. (2015) Journal Modern Physics, 6, 78-87. https://doi.org/10.4236/jmp.2015.61010

[2] Tangherlini, F.R. (2015) Journal Modern Physics, 6, 1360-1370. https://doi.org/10.4236/jmp.2015.69141

[3] Tangherlini, F.R. (2016) Journal Modern Physics, 7, 1829-1844. https://doi.org/10.4236/jmp.2016.713163

[4] Tangherlini, F.R. (2017) Journal Modern Physics, 8, 622-635. https://doi.org/10.4236/jmp.2017.84042

[5] Tangherlini, F.R. (2018) Journal Modern Physics, 9, 573-583. https://doi.org/10.4236/jmp.2018.94039

[6] Ade, P.A.R., et al. (2016) Planck Collaboration: Astronomy and Astrophysics, 594, A13.

[7] Riess, A.G., et al. (2016) SHOES Collaboation: Astrophysical Journal, 826, 56. https://doi.org/10.3847/0004-637X/826/1/56

[8] Shadab, A., et al. (2016) BOSS Collaboration: Monthly Notices Royal Astronomical Society, 000, 1-38.

[9] Riess, A.G., et al. (1998) Astronomical Journal, 116, 1009-1038. https://doi.org/10.1086/300499

[10] Schmidt, B., et al. (1998) Astrophysical Journal, 507, 45-63. https://doi.org/10.1086/306308

[11] Perlmutter, S., et al. (1999) Astrophysical Journal, 517, 565-586. https://doi.org/10.1086/307221

[12] Costa, E., et al. (1997) Nature (London), 387, 783-785. https://doi.org/10.1038/42885

[13] Metzger, M.R., et al. (1997) Nature (London), 387, 878-880. https://doi.org/10.1038/43132

[14] Frail, D.A., et al. (1997) Nature (London), 389, 261-263. https://doi.org/10.1038/38451

[15] Riess, A.G., et al. (2001) Astrophysical Journal, 560, 49-71. https://doi.org/10.1086/322348

[16] Verde, L., et al. (2002) Monthly Notices Royal Astronomical Society, 335, 432-440. https://doi.org/10.1046/j.1365-8711.2002.05620.x

[17] Hawkins, E., et al. (2003) Monthly Notices Royal Astronomical Society, 346, 78-96. 
https://doi.org/10.1046/j.1365-2966.2003.07063.x

[18] Anderson, L., et al. (2012) Monthly Notices Royal Astronomical Society, 427, 3435-3457. https://doi.org/10.1111/j.1365-2966.2012.22066.x

[19] Anderson, L., et al. (2014) Monthly Notices Royal Astronomical Society, 439, 83-101. https://doi.org/10.1093/mnras/stt2206

[20] Agostini, M., et al. (2017) Borexino Collaboration: Astroparticle Physics, 86, 1-17.

[21] Achteberg, A., et al. (2007) Astrophysical Journal, 664, 397-410. https://doi.org/10.1086/518596

[22] Vieregg, A.G., et al. (2011) Astrophysical Journal, 736, 50. https://doi.org/10.1088/0004-637X/736/1/50

[23] Adrian-Martinez, S., et al. (2013) Astronomy \& Astrophysics, 559, A9. https://doi.org/10.1051/0004-6361/201322169

[24] Adrian-Martinez, S., et al. (2017) European Physical Journal C, 77, 20. https://doi.org/10.1140/epjc/s10052-016-4496-8

[25] Avrorin, A., et al. (2011) Physics of Particles and Nuclei Letters, 8, 704-716. https://doi.org/10.1134/S154747711107003X

[26] Fukuda, S., et al. (2002) Astrophysical Journal, 578, 317-324. https://doi.org/10.1086/342405

[27] Aharmim, B., et al. (2014) Astroparticle Physics, 55, 1-76. https://doi.org/10.1016/j.astropartphys.2013.12.004

[28] Asakura, K., et al. (2015) Astrophysical Journal, 806, 87. https://doi.org/10.1088/0004-637X/806/1/87

[29] Kochkarov, M.M., et al. (2015) Physics of Particles and Nuclei, 46, 197-200. https://doi.org/10.1134/S1063779615020124

[30] Abassi, R., et al. (2012) Nature (London), 484, 351-354. https://doi.org/10.1038/nature11068

[31] Aartsen, M.G., et al. (2013) IceCube Collaboration: Physical Review Letters, 111, Article ID: 021103.

[32] Bahcall, J.N. (1976) Redshifts as Distance Indicators. In: Field, G.B., Bahcall, J.N. and Arp, H., Eds., The Redshift Controversy, W. A. Benjamin, Reading, 61-121.

[33] Arp, H. (1966) Astrophysical Journal Supplement, 14, 1-20.

[34] Abbott, B.P., et al. (2017) LIGO Scientific Collaboration and Virgo Collaboration, Astrophysical Journal Letters, 848, L12. https://doi.org/10.3847/2041-8213/aa91c9

[35] Abbott, B.P., et al. (2017) LIGO Scientific Collaboration and Virgo Collaboration, Physical Review Letters, 119, Article ID: 161101. https://doi.org/10.1103/PhysRevLett.119.161101

[36] Goldstein, A., et al. (2017) Fermi-GBM, Astrophysical Journal Letters, 848, L14. https://doi.org/10.3847/2041-8213/aa8f41

[37] Savchenko, V., et al. (2017) INTEGRAL Collaboration, Astrophysical Journal Letters, 848, L15. https://doi.org/10.3847/2041-8213/aa8f94

[38] Agostini, M., et al. (2017) Borexino Collaboration, Astrophysical Journal, 850, 21. https://doi.org/10.3847/1538-4357/aa9521 